\section{Introdução da lactação e amenorréia como método contraceptivo (LAM) em um programa de planejamento familiar pós- parto: repercussões sobre a saúde das crianças}

\section{Introduction of lactational amenorrhea as a contraceptive method (LAM) in a post- partum family planning program: outcomes on children's health}

José Guilherme Cecatti 1

Ademilza Siqueira Araújo 2

Maria José Osis 3

Luiz Carlos Santos 4

Aníbal Faúndes 5

1,5 Centro de Pesquisas Materno-Infantis de Campinas. Departamento de Tocoginecologia. Universidade Estadual de Campinas. Rua Alexander Fleming, 101. Campinas, SP, Brasil. CEP: $13.083-881$

1-3,5 Centro de Pesquisas Materno-Infantis de Campinas. Campinas, SP, Brasil

${ }^{4}$ Instituto Materno Infantil de Pernambuco, IMIP, Recife, PE, Brasil

\begin{abstract}
Objectives: to assess the effect of lactation introduction and amenorrhea as a contraceptive method on child health in a post-delivery program.

Methods: operational survey assessing total and exclusive breast feeding and some health indicators of children up to one year old, before and after the LAM program as a method offered to women after delivery in addition to education on breast feeding and family planning. Six hundred and ninety eight women who had their prenatal care in the Institution were broken into two groups: Group A receiving assistance before and $B$ receiving assistance following the program. Statistical analysis used the Chi-Square test, survival analysis and Cox's logistic regression.

Results: Group A had $85.6 \%$ of morbid episodes up to 12 months and Group B 71.2\%. The number of hospital admissions more than doubled for children in Group A. Weight and height of the children at twelve months old and accumulated rates of total breast feeding were significantly higher in Group $B$.

Conclusions: this educational program is associated to better child health results and can be used in other institutions for its beneficial impact on child healthcare.

Key words Breast feeding, Family Planning Services, Puerperium, Contraception, Child Health (Public Health)
\end{abstract}

\section{Resumo}

Objetivos: avaliar o efeito da introdução da lactação e amenorréia como método anticoncepcional sobre a saúde da criança em um programa puerperal.

Métodos: pesquisa operacional que avalia a duração do aleitamento materno total e exclusivo, $e$ alguns indicadores de saúde das crianças até um ano de idade, antes e após a introdução da LAM como método oferecido às mulheres no pós-parto, com reforços educativos sobre amamentação e planejamento familiar. Foram incluídas 698 mulheres que tiveram pré-natal e parto no Instituto Materno Infantil de Pernambuco divididas em: Grupo A, que recebeu assistência antes e B, após a intervenção. $\mathrm{Na}$ análise estatística utilizaram-se o teste Qui-Quadrado, análise de sobrevivência e regressão logística de Cox.

Resultados: o Grupo A teve 85,6\% de episódios mórbidos até 12 meses e o Grupo B 72,1\%. O número de internações foi mais que o dobro para as crianças do Grupo A. O peso, a estatura das crianças aos 12 meses e as taxas acumuladas de aleitamento materno total foram significativamente maiores no Grupo B.

Conclusões: esta intervenção educacional associa-se a melhores resultados infantis e pode ser aplicada em outros serviços também pelos benefícios à saúde das crianças.

Palavras-chave Aleitamento materno, Serviços de Planejamento Familiar, Puerpério, Anticoncepção, Saúde infantil (Saúde Pública) 


\section{Introdução}

Embora antigo, o conhecimento sobre o papel anticoncepcional do aleitamento materno é relativamente pouco difundido e utilizado. ${ }^{1,2} \mathrm{Na}$ prática, o aleitamento materno e o planejamento familiar atuam conjuntamente promovendo a sobrevivência infantil, uma vez que o estímulo à amamentação exclusiva induz a um aumento do intervalo intergestacional e propicia a manutenção da lactação até os seis meses de vida, período no qual há a introdução de outros alimentos à dieta infantil. Assim, o aleitamento materno e o planejamento familiar são medidas claras de saúde preventiva, necessitando ambos de apoio, através de programas que os incentivem, trazendo com isso benefícios inquestionáveis para a saúde da mãe e da criança. ${ }^{3}$

Alguns estudos têm demonstrado ainda que um intervalo de dois ou mais anos entre nascimentos leva a uma redução significativa na morbimortalidade infantil e também uma redução na morbidade materna, principalmente nos países menos desenvolvidos. 4 Dados demográficos revelaram ainda que, em muitos países em desenvolvimento, a amamentação, por si só, fornece proteção contra gravidez maior que a oferecida por outros métodos reversíveis de anticoncepção. 3,4

A maternidade é um local adequado para se revisar ou ensinar às mães os conceitos básicos sobre o início e a manutenção da amamentação, já abordados durante o acompanhamento pré-natal. A mensagem de que o aleitamento exclusivo promove uma proteção contra uma nova gestação até o retorno da menstruação deve ser fornecida nessa ocasião, permitindo postergar a utilização de outros métodos contraceptivos nos primeiros meses pós-parto. A utilização do método contraceptivo da lactação e amenorréia (LAM) no pós-parto é uma alternativa a mais de escolha informada, sobretudo quando outros métodos anticoncepcionais não são de fácil acesso ou não têm boa aceitação. Entretanto, o conhecimento sobre a aplicação desse efeito fisiológico não tem sido explorado, nem tampouco utilizado sistematicamente na anticoncepção puerperal. ${ }^{5}$

Apesar dos benefícios do aleitamento materno sobre a saúde da criança serem universalmente reconhecidos, e também os efeitos da lactação natural sobre a fertilidade comprovados, existe algum ceticismo sobre o seu uso como um método anticoncepcional. ${ }^{4}$ No ano de 1988, em Bellagio, Itália, um grupo de cientistas reuniu-se e, através de uma Declaração de Consenso, recomendou a introdução da LAM como um método de planejamento familiar, ${ }^{4}$ posteriormente referendado em outra reunião face às novas evidências científicas disponíveis. 6

A LAM tem como princípios, além do estado de amenorréia das mulheres, a amamentação exclusiva dos seus filhos e a idade máxima de seis meses para o bebê. Nessas condições, a probabilidade acumulada de ocorrer uma nova gravidez nesse período é menor do que 2\%.6-11 Além disso, estudos demográficos sugerem que, em países em desenvolvimento, as taxas de gravidezes entre mulheres lactantes amenorreicas com mais de seis meses pós-parto é menor que $10 \% .4$

Realizou-se um estudo multicêntrico em dez diferentes populações com a finalidade de avaliar a aceitabilidade, satisfação e eficácia da LAM em um programa de planejamento familiar. Confirmou-se que é um método anticoncepcional com boa aceitação, se adequadamente orientado.12,13 Também se confirmou sua eficácia, de $98 \%$ de proteção contra uma nova gestação, ao final de seis meses pósparto. 14

Nenhum estudo foi realizado para se avaliar a efetividade de incluir a LAM em um programa de planejamento familiar pós-parto para mulheres de baixa renda, e analisar os efeitos não só com relação à fertilidade, mas também os benefícios dessa estratégia sobre a saúde da mulher e da criança, apesar de todas as suposições que assim o indicam.

A comprovação da eficácia da LAM como método anticoncepcional nos primeiros seis meses pósparto já é consenso em todo o mundo. O incentivo ao aleitamento materno, pelos benefícios inquestionáveis sobre a saúde da criança nos primeiros seis meses de vida, é uma prática que todos os profissionais de saúde devem estimular, tanto na promoção da saúde das crianças quanto das mães, através do aumento do intervalo intergestacional e da não-utilização de métodos anticoncepcionais quando esses ainda não são necessários, podendo interferir no sucesso da amamentação. Uma forma de atingir este objetivo é oferecendo a LAM como opção anticoncepcional nos primeiros seis meses pós-parto. Como no Brasil as mulheres amamentam em média por pouco tempo e a taxa de morbimortalidade infantil é elevada, 15 a adoção de medidas que incentivem o aleitamento materno contribuiria para a redução da morbimortalidade infantil, através do aumento da duração do aleitamento materno, tanto o exclusivo quanto o parcial.

O único estudo nacional avaliando a LAM foi realizado no Instituto Materno Infantil de Pernambuco, 16 do qual se originam os dados ora apresentados. Esse estudo implementou uma intervenção que incluía a introdução da LAM como um método anticoncepcional adicional ao programa de planejamen- 
to familiar pós-parto, além de uma estratégia de reforço ao aleitamento materno e ao espaçamento das gravidezes, tanto durante o pré-natal quanto durante a internação para o parto e, posteriormente, nas visitas de puerpério.

Assim, caso fossem observados efeitos positivos sobre a saúde das crianças, após essa estratégia educativa com a introdução da LAM como opção anticoncepcional adicional no planejamento familiar pós-parto utilizada no estudo, estaria clara a efetividade de se estimular e incentivar programas semelhantes em outras instituições. A busca dessa evidência é a justificativa principal desse trabalho. Os objetivos do presente estudo foram avaliar a duração do aleitamento materno, total e exclusivo, e alguns indicadores de saúde das crianças até um ano de idade, comparativamente entre mulheres que receberam orientação anticoncepcional tradicional (pré-intervenção) e as que participaram de um novo programa de orientação (pós-intervenção) que incluiu a introdução da LAM como uma nova opção anticoncepcional.

\section{Métodos}

O presente estudo é parte de uma pesquisa maior sobre LAM.16 Tratou-se de um estudo operacional semi-experimental, utilizando duas séries temporais. 17

Inicialmente foram incluídas mulheres que receberam a orientação tradicional dada pelo Instituto Materno Infantil de Pernambuco (IMIP), sobre amamentação e contracepção puerperal (Grupo A). O IMIP é um grande hospital materno infantil localizado na cidade de Recife, estado de Pernambuco, no Nordeste do Brasil, que oferece serviços médicos terciários a mulheres e crianças de baixa renda do município e Região, e que tradicionalmente tem estimulado todas as práticas favorecedoras do aleitamento materno como condição adjuvante da saúde materna e infantil.

O estudo incluiu duas coortes de mulheres atendidas durante o parto na Maternidade do referido serviço, no período de julho de 1992 a outubro de 1994. A primeira coorte foi constituída por mulheres que receberam a orientação anticoncepcional tradicionalmente adotada no serviço (Grupo A), a qual incluía a orientação quanto ao uso de métodos comportamentais, de barreira, hormonais orais e injetáveis, além de dispositivos intra-uterinos. A segunda coorte (Grupo B) foi composta por mulheres que receberam a intervenção do estudo, orientação reforçada acerca do aleitamento materno e contracepção, incluindo a LAM como mais uma opção contraceptiva. O grupo A incluiu 350 mulheres que atendiam os seguintes requisitos: terem realizado pelo menos uma consulta pré-natal no IMIP, terem retornado à consulta no oitavo dia pós-parto, morarem em Recife ou na área metropolitana da cidade, não terem tido gêmeos ou natimorto no último parto, não apresentarem fator materno-infantil que inviabilizasse o aleitamento, não terem feito ligadura tubária ou histerectomia nos primeiros seis meses pós-parto, e terem voltado a ter relações sexuais nos primeiros seis meses pós-parto. Foram entrevistadas em uma única ocasião, cerca de um ano pósparto, por entrevistadoras treinadas.

No grupo A, através de um levantamento retrospectivo, as mulheres que preenchiam os pré-requisitos para o estudo eram convidadas, através de uma carta, a comparecer ao ambulatório de ginecologia do IMIP, trazendo consigo o seu último filho, oferecendo-se a ambos consultas respectivamente na ginecologia e pediatria. Após a consulta, a mulher era convidada a participar do estudo. Caso aceitasse, era entrevistada imediatamente por uma das entrevistadoras. Essas entrevistas aconteceram quando a criança tinha 12 meses de idade, já que o objetivo era comparar a saúde infantil ao final do primeiro ano de vida

Fizeram parte do grupo B, 350 mulheres que atendiam os mesmos pré-requisitos do grupo A e ainda: desejassem evitar filhos pelo menos nos primeiros seis meses pós-parto, e não tivessem autorização para fazer ligadura tubária. Os sujeitos do Grupo B eram identificados na consulta de oitavo dia pós-parto. Nessa ocasião, as mulheres recebiam uma palestra sobre aleitamento materno e anticoncepção, explicando sobre o uso da LAM como opção anticoncepcional entre outros métodos.

As que cumpriam os requisitos eram agendadas para a consulta de $40^{\circ}$ dia pós-parto. Nessa ocasião elas ouviam mais uma palestra educativa e, em seguida eram convidadas a participar do estudo. Quando a mulher aceitava, a sua admissão no estudo fazia-se imediatamente, realizando-se a primeira entrevista e agendando-se a seguinte para três meses pós-parto. Na seqüência, agendavam-se novas entrevistas para os seis, nove e doze meses pós-parto. Quando uma mulher não comparecia na data marcada, enviava-se uma carta-lembrete. As entrevistas eram realizadas em todos os retornos pré-fixados até um ano, para esse grupo.

As mulheres do Grupo B que optavam pela LAM como método anticoncepcional recebiam um prospecto de orientação com informações sobre a LAM como método e um cartão com recomendações sobre ela. Essas mulheres eram instruídas sobre co- 
mo proceder se tivessem qualquer dúvida em relação à amamentação e anticoncepção. Recebiam fichas telefônicas e o número de um telefone, através do qual poderiam entrar em contato rapidamente com o pessoal do estudo para resolver suas dúvidas.

As mulheres admitidas no estudo, nos dois grupos, eram orientadas a trazerem consigo o seu último filho nascido na Maternidade do IMIP, para que ele fosse examinado por um pediatra, com a finalidade de se avaliar sua condição de saúde e desenvolvimento pondero-estatural. Para a variável independente "tipo de orientação sobre amamentação e anticoncepção no puerpério", estudaram-se as seguintes variáveis dependentes: amamentação, tempo de aleitamento materno total, morbidade referida pela mãe do último filho até 12 meses (qualquer episódio da criança "ter ficado doente" referido pela mãe), óbito do último filho, hospitalização do último filho até 12 meses, desenvolvimento ponderal e estatural da criança aos 12 meses.

Os resultados foram controlados pela idade materna, escolaridade, número de gravidezes, estado marital, número de abortos, número de nascidos vivos, número de filhos vivos, amamentação do filho anterior, razões para o desmame do filho anterior e tipo do último parto.

Foram considerados para a comparabilidade das informações no presente estudo apenas os dados obtidos da última entrevista no grupo B, no que se refere à saúde das crianças. Na avaliação dos episódios mórbidos das crianças, foram feitos agrupamentos de causas, para a realização da análise através da comparação da proporção dos eventos mórbidos entre os dois grupos. 18

Inicialmente as variáveis foram estudadas por grupos, e as comparações foram feitas através do teste Qui-Quadrado para variáveis qualitativas, QuiQuadrado para tendência para as variáveis categóricas ordenadas e teste $t$ de Student para variáveis quantitativas. Em seguida, realizaram-se uma análise de sobrevivência para as variáveis aleitamento materno exclusivo e total (onde o evento foi o término do aleitamento materno exclusivo e total, respectivamente), e uma regressão logística de Cox para os eventos mórbidos e hospitalização das crianças. ${ }^{19}$ As taxas acumuladas de aleitamento foram calculadas pelo método de tabela de vida (intervalo mensal) e os dois Grupos comparados através da estatística de Wilcoxon-Gehan. Foram censurados os casos de perda de seguimento entre dois controles consecutivos, computando-se para cálculo da taxa seguinte metade das censuras para compor o denominador de expostos ao risco do evento.

$\mathrm{O}$ projeto de pesquisa foi previamente aprovado pela Comissão de Ética do IMIP de Recife. Na época em que o projeto foi desenvolvido ainda não estava em vigor a resolução 186/96 do Ministério da Saúde que regulamenta a participação de seres humanos em pesquisas. O consentimento das mulheres para participarem da pesquisa foi oral, visto que nenhuma intervenção médica propriamente dita estava programada.

\section{Resultados}

No estudo original, na consulta de 40 dias pós-parto $4,9 \%$ das mulheres do grupo B haviam aceitado a LAM como método contraceptivo, e 58,8\% das mulheres que estavam em amenorréia e em aleitamento exclusivo disseram que não estavam usando nenhum método anticoncepcional; $1 \%$ escolheu a pílula; $6 \%$ condom e $12 \%$ coito interrompido. A LAM teve uma prevalência de $13 \%$ aos três meses pós-parto no grupo B e quase $6 \%$ dessas mulheres continuavam usando esse método aos seis meses pós-parto. A proporção de mulheres que tiveram uma nova gestação um ano após o parto foi $50 \%$ mais baixa no grupo pós-intervenção.

Observou-se, neste estudo, que a porcentagem de mulheres que não estavam usando nenhum método contraceptivo um ano após o parto foi significativamente mais baixa após a intervenção do que antes (respectivamente $7,4 \%$ e $17,7 \%, p<0,05$ ). Também a proporção de gravidezes com um ano pós-parto, mostrou uma diferença significativa entre os Grupos, de 7,4\% no Grupo com a nova abordagem e $14,3 \%$ no Grupo anterior a ela.

Considerando que houve uma perda de seguimento de 38,2\% no grupo B aos 12 meses pós-parto, compararam-se algumas variáveis das mulheres que compareceram a essa entrevista $(n=215)$ com as que não compareceram $(\mathrm{n}=133)$. A análise dessa comparação com relação à idade materna, número de gravidezes, amamentação do filho anterior e tempo de amamentação do filho anterior não mostrou diferenças significativas entre os grupos, indicando que a perda de seguimento parece não ter sido seletiva.

A avaliação dos dois grupos, segundo algumas características como escolaridade, estado marital, número de nascidos vivos e filhos vivos, número de abortos e tipo de parto, 16 não demonstrou diferenças estatisticamente significantes entre ambos.

Houve um predomínio de maior idade e maior número de gravidezes entre as do grupo $\mathrm{A}$, mas não houve diferença quanto à experiência prévia de amamentação (Tabela 1). A quase totalidade das mulheres amamentou seus filhos na atual gravidez, em ambos 
os grupos. No grupo A, cinco crianças não foram amamentadas, uma porque faleceu, duas porque não "pegaram o seio" e duas porque as mães estavam doentes. Já no grupo B, das três crianças que não foram amamentadas, uma "não pegou o seio", uma estava doente e uma a mãe "não tinha leite". Ocorreram dois óbitos de crianças do grupo A até os 12 meses e nenhum óbito para o grupo B. Além disso, a morbidade das crianças do grupo A foi significativamente maior do que do grupo B (Tabela 2).

\section{Tabela 1}

Distribuição percentual das mulheres por idade, número de gravidezes e amamentação prévia segundo grupos de intervenção.

\begin{tabular}{|c|c|c|c|c|c|}
\hline \multirow{2}{*}{ Variáveis } & \multicolumn{2}{|c|}{ Grupo B } & \multicolumn{2}{|c|}{ Grupo B } & \multirow{2}{*}{$p$} \\
\hline & $\mathrm{n}$ & $\%$ & $\mathrm{n}$ & $\%$ & \\
\hline Idade (anos) & & & & & $<0,001$ \\
\hline $14-19$ & 49 & 14,0 & 97 & 27,9 & \\
\hline $20-24$ & 128 & 36,6 & 133 & 38,2 & \\
\hline $25-29$ & 92 & 26,3 & 63 & 18,1 & \\
\hline 30 ou mais & 81 & 23,1 & 55 & 15,8 & \\
\hline Gravidezes & & & & & 0,002 \\
\hline 1 & 145 & 41,4 & 188 & 54,0 & \\
\hline 2 & 109 & 31,1 & 86 & 24,7 & \\
\hline$\geq 3$ & 96 & 27,4 & 74 & 21,3 & \\
\hline Total de mulheres & 350 & 100,0 & 348 & 100,0 & \\
\hline Amamentaram antes & & & & & 0,330 \\
\hline Sim & 121 & 89,6 & 102 & 88,7 & \\
\hline Não & 14 & 10,4 & 13 & 11,3 & \\
\hline Total de mulheres* & 135 & 100,0 & 115 & 100,0 & \\
\hline
\end{tabular}

*Excluídas as primíparas

Tabela 2

Morbidade e mortalidade das crianças, até os 12 meses pós-parto, segundo grupos de intervenção.

\begin{tabular}{|c|c|c|c|c|}
\hline \multirow{2}{*}{ Saúde perinatal } & \multicolumn{2}{|c|}{ Grupo B } & \multicolumn{2}{|c|}{ Grupo B } \\
\hline & $n$ & $\%$ & $\mathrm{n}$ & $\%$ \\
\hline \multicolumn{5}{|l|}{ Mortalidade } \\
\hline Sim & 2 & 0,6 & - & . \\
\hline Não & 348 & 99,4 & 215 & 100,0 \\
\hline Total de mulheres & 350 & 100,0 & $215^{* *}$ & 100,0 \\
\hline \multicolumn{5}{|l|}{ Morbidade $* * *$} \\
\hline Sim & 298 & 85,6 & 155 & 72,1 \\
\hline Não & 50 & 14,4 & 60 & 27,9 \\
\hline Total de mulheres & $348 *$ & 100,0 & $215^{* *}$ & 100,0 \\
\hline
\end{tabular}

* Faltam informações de dois casos ; * Faltam informações de 133 casos para o $12^{\circ}$ mês; *** $\chi^{2}=15,50 p<0,001$ 
A Tabela 3 mostra que o maior número de episódios mórbidos entre as crianças do grupo A deveu-se basicamente a doenças infecciosas. No período de 12 meses após o parto, 18,1\% das crianças do grupo A tiveram alguma internação hospitalar, significativamente maior que os $7,1 \%$ do grupo $\mathrm{B}$. Tanto o peso como a estatura das crianças aos 12 meses foram significativamente maiores para o grupo B (Tabela 4).
Aos seis meses pós-parto aproximadamente $45 \%$ das mulheres no grupo A e quase $53 \%$ no grupo B ainda amamentavam, e os valores foram significativamente maiores para o grupo B ao longo dos 12 meses de acompanhamento. As medianas de amamentação foram de 5,2 meses no grupo A e de 6,5 meses no grupo B (Tabela 5 e Figura 1).

Tabela 3

Episódios mórbidos do filho incluído no estudo até os 12 meses pós-parto, segundo grupos de intervenção.

\begin{tabular}{|c|c|c|c|}
\hline \multirow{2}{*}{ Episódios mórbidos* } & \multirow{2}{*}{$\begin{array}{l}\text { Grupo A } \\
n=348\end{array}$} & \multirow{2}{*}{$\begin{array}{l}\text { Grupo B } \\
n=215\end{array}$} & \multirow{2}{*}{$p$} \\
\hline & & & \\
\hline Diarréia/infecção intestinal/desidratação & 87 & 38 & 0,042 \\
\hline Doenças imunopreviníveis (catapora, rubéola, sarampo, coqueluche) & 18 & 6 & 0,174 \\
\hline Infecções de vias aéreas superiores/otite & 304 & 111 & $<0,001$ \\
\hline Pneumonia/broncopneumonia & 12 & 6 & 0,666 \\
\hline Outras infecções (sistêmica/urinária) & 13 & 2 & 0,044 \\
\hline Alergia/afecções na pele & 52 & 25 & 0,266 \\
\hline Anemia/desnutrição & 12 & 7 & 0,902 \\
\hline $\begin{array}{l}\text { Sinais e sintomas mal definidos (febre, convulsão, problema renal, cist } \\
\text { problema cirúrgico, cardíaco, icterícia, cólicas, etc.) }\end{array}$ & 80 & 27 & 0,002 \\
\hline Outros & 19 & 12 & 0,950 \\
\hline
\end{tabular}

*Total não corresponde à soma dos episódios mórbidos

Peso e estatura do filho incluído no estudo até os 12 meses pós-parto, segundo grupos de intervenção.

\begin{tabular}{|c|c|c|c|c|c|}
\hline & \multicolumn{2}{|c|}{ Grupo B } & \multicolumn{2}{|c|}{ Grupo B } & \multirow{2}{*}{$p$} \\
\hline & $\mathrm{n}$ & $\%$ & $\mathrm{n}$ & $\%$ & \\
\hline Peso aos 12 meses & & & & & 0,006 \\
\hline De $5 \mathrm{~kg}$ até $9 \mathrm{~kg}$ & 80 & 23,5 & 20 & 11,3 & \\
\hline Mais de $9 \mathrm{~kg}$ até $12 \mathrm{~kg}$ & 232 & 68,2 & 141 & 79,7 & \\
\hline Mais de $12 \mathrm{~kg}$ & 28 & 8,2 & 16 & 9,0 & \\
\hline Total de mulheres & $340 *$ & 100,0 & $177 * *$ & 100,0 & \\
\hline Estatura aos 12 meses & & & & & 0,090 \\
\hline 61 a $70 \mathrm{~cm}$ & 61 & 19,7 & 28 & 16,0 & \\
\hline 71 a $80 \mathrm{~cm}$ & 240 & 77,4 & 136 & 77,7 & \\
\hline $81 \mathrm{~cm}$ ou mais & 9 & 2,9 & 11 & 6,3 & \\
\hline Total de mulheres & $310 * * *$ & 100,0 & $175 * * *$ & 100,0 & \\
\hline
\end{tabular}

* Falta informação de 10 mulheres; ** Falta informação de 38 mulheres; *** Falta informação de 40 mulheres 


\section{Tabela 5}

Taxas acumuladas de continuação de aleitamento materno total do último filho segundo grupo.

\begin{tabular}{|c|c|c|}
\hline \multirow{2}{*}{ Tempo (mês) } & Grupo A & Grupo B \\
\hline & $\%$ & $\%$ \\
\hline $0 \begin{array}{lll}0 & -I & 1\end{array}$ & 98,6 & 99,1 \\
\hline $1-12$ & 87,1 & 92,9 \\
\hline $2-13$ & 76,6 & 77,5 \\
\hline $3-14$ & 60,6 & 65,3 \\
\hline $4-15$ & 51,1 & 57,3 \\
\hline $5-16$ & 45,4 & 52,8 \\
\hline $6-17$ & 38,0 & 47,4 \\
\hline $7 \quad-18$ & 32,6 & 41,6 \\
\hline $8-19$ & 28,3 & 37,1 \\
\hline $9-10$ & 26,9 & 34,1 \\
\hline $10-\mid 11$ & 24,9 & 31,8 \\
\hline $11-12$ & 24,6 & 30,9 \\
\hline Mediana (meses) & 5,2 & 6,5 \\
\hline (n) & $(350)$ & $(348)$ \\
\hline
\end{tabular}

$p=0,023$ (teste de Wilcoxon - Gehan)

Figura 1

Taxa acumulada de continuação de aleitamento materno total (\%) até os 12 meses, segundo os grupos de intervenção.

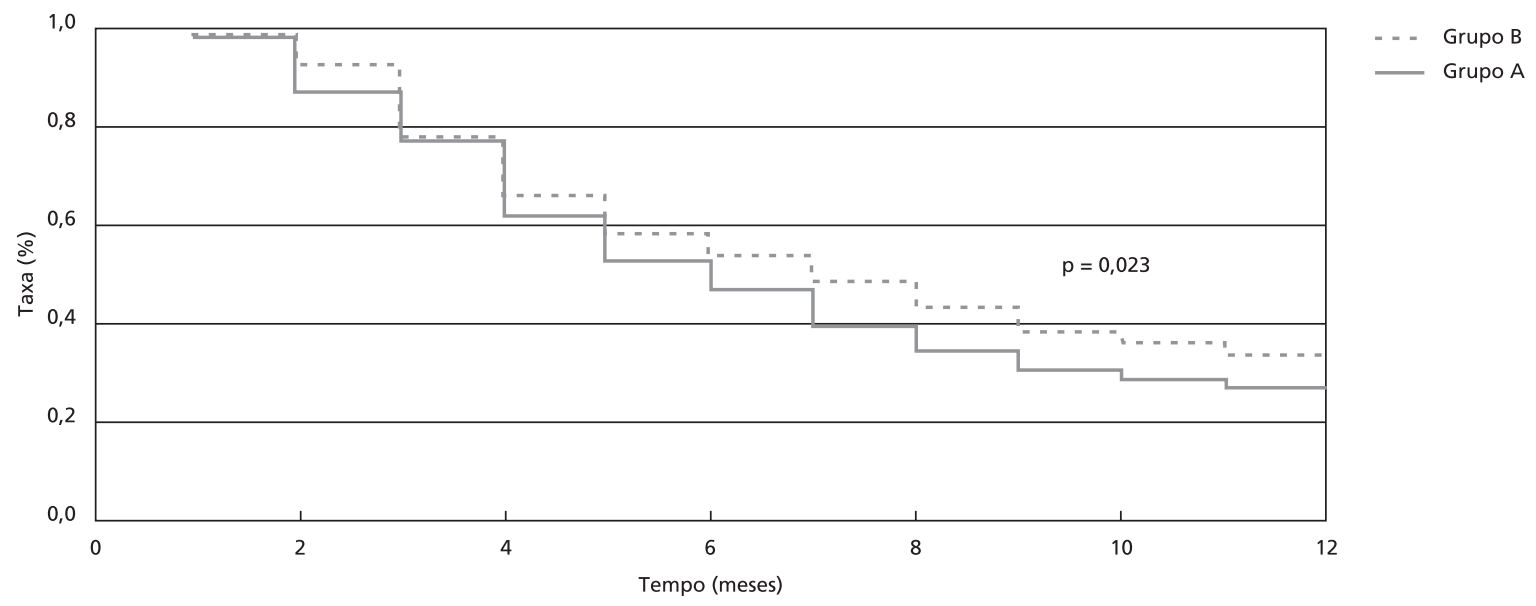


Entretanto, para o aleitamento materno exclusivo ou quase exclusivo, essas taxas foram significativamente maiores para as mulheres do grupo A, ou seja, embora a mediana de tempo de amamentação tenha sido maior para as mulheres do grupo B, elas amamentaram exclusivamente ou quase por um tempo menor (Figura 2). Nos dois modelos de regressão utilizados, tendo como variável dependente a morbidade da criança e sua hospitalização até 12 meses, a única variável preditora, dentre todas as utilizadas que entraram para o modelo, que foi identificada como estatisticamente associada a essas ocorrências foi pertencer ao grupo A, ou seja, a intervenção de um reforço educacional em estimular o aleitamento materno e em métodos anticoncepcionais no puerpério, incluindo a LAM entre eles, foi a única variável que se associou à menor morbidade e ao menor número de hospitalizações das crianças até um ano de vida.

\section{Figura 2}

Taxas acumuladas de continuação de aleitamento materno quase exclusivo (\%) até os seis meses, segundo os grupos de intervenção.

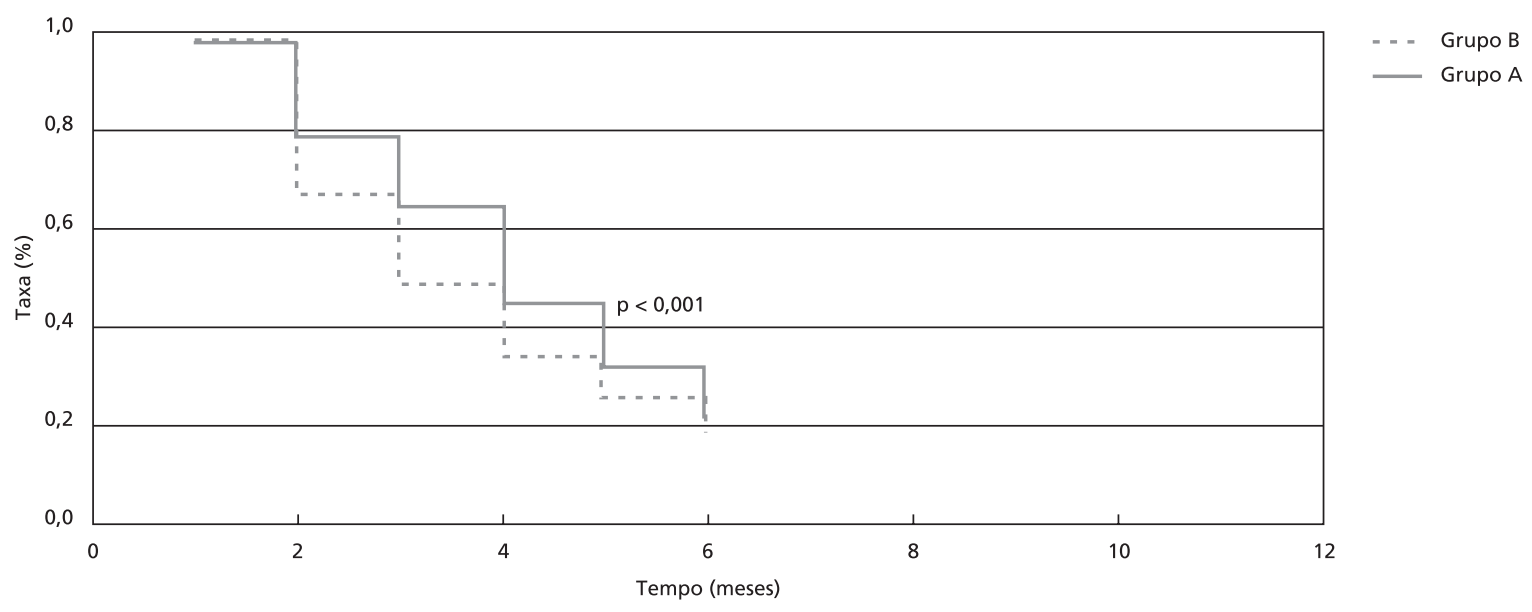

\section{Discussão}

Esse estudo foi realizado com o objetivo de se avaliar a influência da LAM introduzida em um programa de planejamento familiar pós-parto, sobre a saúde das crianças das mulheres submetidas à nova estratégia de orientação anticoncepcional, priorizando a manutenção do aleitamento materno em uma população de baixa renda no Nordeste do Brasil, região com altas taxas de morbimortalidade infantil.

Os resultados obtidos mostraram que a ocorrência de episódios mórbidos até 12 meses pós-parto, principalmente de causas infecciosas, e de internações hospitalares foi significativamente menor no grupo de mulheres submetidas a essa intervenção educacional (Grupo B). O peso e a estatura dessas crianças também foram significativamente superiores em relação ao grupo anterior à intervenção (Grupo A). Esses dados são ainda coerentes com uma taxa acumulada de aleitamento materno total aos 12 meses pós-parto estatisticamente superior no grupo B.

Nos dois grupos a faixa etária predominante foi entre 20 e 24 anos; entretanto, o grupo pós-intervenção teve quase o dobro de adolescentes em relação ao grupo anterior à intervenção. Uma possível explicação refere-se à maneira como, no desenho do estudo, selecionaram-se as puérperas do Grupo A, retrospectivamente, algum tempo após o parto, durante algum retorno à instituição. Era de se esperar, pelas características sociais e dificuldades práticas que enfrentam, que as adolescentes retornassem 
menos ao serviço; portanto, com menor probabilidade de serem selecionadas para ingressar no estudo. Houve uma tendência significativa das mulheres do Grupo B terem um menor número de gravidezes, em concordância também com o maior número de mulheres com menor idade. Embora tenha sido observado que entre mulheres com maior número de filhos ocorrem as maiores taxas de lactação prolongada, ${ }^{20,21}$ nesta pesquisa notou-se que o número de filhos vivos foi semelhante entre os dois grupos. Os estudos mostraram, contudo, que a educação sanitária é mais efetiva em aumentar as taxas de início de amamentação, ou seja, das mulheres começarem a amamentar seus filhos entre mães primíparas. ${ }^{20} \mathrm{Em}$ ambos os grupos, $90 \%$ das mulheres que tinham mais de um filho, possuíam experiência prévia de amamentação do filho anterior, e o tempo de amamentação desse filho não mostrou diferença entre os grupos.

Com relação aos episódios mórbidos da criança até 12 meses pós-parto, o grupo A apresentou 85,6\% de morbidade e o grupo B $72,1 \%$, mostrando uma diferença significativa entre os grupos. Houve um predomínio de doenças infecciosas nas crianças do grupo A, coerente com uma maior taxa de aleitamento total encontrada no grupo B. Tais resultados reforçam as propriedades antiinfecciosas do leite humano, dando proteção contra doenças infecciosas, particularmente a diarréia.22 Além disso, o número de hospitalizações foi mais que o dobro para as crianças do grupo A e quase dois terços dessas crianças tinham menos de seis meses, enquanto no grupo B essa proporção foi menor que um quinto.

No presente estudo, através de análise multivariada, esteve o grupo A associado à maior internação e morbidade da criança até os 12 meses. Essa associação manteve-se coerente com a maior duração do aleitamento materno. A média de peso e altura das crianças aos 12 meses pós-parto também foi significativamente maior no grupo B, realçando os benefícios do aleitamento materno sobre os indicadores de saúde das crianças. A taxa acumulada de aleitamento materno total, obtida pela análise de sobrevivência, mostrou aos 12 meses $29,4 \%$ para o grupo pós-intervenção e $24,6 \%$ para o grupo pré-intervenção, diferença essa estatisticamente significativa entre os dois grupos, porém baixa quando comparada aos dados da Pesquisa Nacional sobre Demografia e Saúde (PNDS), em 1996, que mostraram uma taxa de aleitamento total aos 12 meses de $37 \% .23$

Nos modelos de regressão utilizados, tendo como variável dependente a morbidade e a hospitalização das crianças até 12 meses, a única variável que foi identificada como estatisticamente associada a essas ocorrências foi pertencer ao grupo A. Isso significa que a diferença básica entre eles, ter recebido a intervenção com a orientação anticoncepcional dirigida ao puerpério, com ênfase para a importância da amamentação à saúde da criança, seu efeito anticoncepcional e a disponibilidade da utilização da LAM como uma opção real, de fato esteve associada à diferença entre os dois grupos, com um melhor desempenho em morbidade e hospitalização das crianças do grupo B, após a intervenção. Ainda que esse processo educativo associado à nova visão da anticoncepção no puerpério não tivesse demonstrado uma menor taxa de gravidezes e uma maior prevalência de utilização de algum método anticoncepcional ao longo do período puerperal, como já relatado em outro artigo específico para esse tópico, 16 esses resultados positivos relativos à saúde da criança ora apresentados seriam mais que suficientes para justificar a adoção desta intervenção em contextos semelhantes para justificar a implementação sistemática de uma abordagem similar em programas de planejamento familiar pós-parto, sobretudo em situações de populações de baixo nível socioeconômico, como é o presente caso.

Os resultados relativos à melhoria encontrada nas taxas de duração do aleitamento materno e os seus benefícios nos indicadores de saúde das crianças são animadores. Eles reforçam os dados de vários estudos que mostraram que o aleitamento materno é uma prática a ser aprendida para ser eficazmente exercida e que populações-alvo respondem favoravelmente à intervenção educacional.13 É bem conhecida a susceptibilidade do comportamento materno para o aleitamento em relação a eventos externos, como campanhas de incentivo e de valorização do leite materno. 24

Apesar dessas mudanças, as taxas de aleitamento materno no Brasil permanecem muito abaixo das recomendações internacionais, mostrando a necessidade de se continuar estimulando a amamentação. 25 Os programas de planejamento familiar pós-parto devem ser voltados para a manutenção da prática do aleitamento materno, onde os métodos anticoncepcionais que venham a interferir na amamentação devem ser proscritos e a LAM deve ser sempre lembrada e ensinada como ser usada eficazmente. É um método universalmente aceito em todo o mundo, pela sua comprovada eficácia e baixo custo.

A proposta original deste estudo não foi a de testar a eficácia da LAM em um programa de planejamento familiar pós-parto, mas a de testar a efetividade de um novo programa de orientação anticoncepcional no pós-parto, com grande ênfase ao aleitamento materno e à possibilidade da utilização 
opcional da LAM como um método eficaz em prevenir a gravidez em período de baixa fertilidade, até o momento que um anticoncepcional de maior eficácia seja realmente necessário. O provável maior benefício dos resultados deste estudo foi demonstrar que foi factível aplicar tal programa a uma população de baixa renda de um país em desenvolvimento, sem prejuízo para a anticoncepção, e com nítidas vantagens em termos de saúde e desenvolvimento pôndero-estatural das crianças. A partir dessa experiência, seria ainda prudente recomendar a aplicação desse conhecimento em populações maiores e diversificadas, em diversas regiões do país, para se ter certeza de sua aplicabilidade geral e uma posterior possível recomendação de utilização de programas semelhantes em todo o território nacional.

Com o presente estudo concluiu-se que a grande maioria das mulheres nos dois grupos iniciou a amamentação, a qual foi mantida por mais tempo no grupo B. Entretanto, a taxa acumulada de continuação do aleitamento exclusivo foi significativamente maior no Grupo A; as crianças do Grupo B apresen- taram significativamente menos episódios mórbidos, associação essa que se manteve pela análise multivariada e é coerente com a maior duração do aleitamento materno total nesse Grupo.

Embora o tamanho da amostra seja pequeno para conclusões acerca de mortalidade, observou-se uma baixa taxa de mortalidade nos dois grupos. A taxa de internação hospital foi significativamente maior no Grupo A em relação ao B, as crianças do Grupo B tiveram médias de peso e altura aos 12 meses pósparto significativamente maiores que as do Grupo A. Não existem estudos publicados que tenham avaliado o efeito da introdução da LAM sobre a saúde infantil em programas de planejamento familiar puerperal da forma como se propôs no presente estudo. Seria já suficiente que não houvesse resultados negativos relativos à saúde infantil para que a recomendação da introdução da LAM pudesse ser feita. Com os resultados favoráveis que o presente estudo mostra, sem nenhum prejuízo também para a saúde materna e a anticoncepção, 16 tal recomendação pode ser feita de maneira ainda mais segura.

\section{Agradecimentos}

O desenvolvimento e implementação do projeto de pesquisa original tiveram financiamento do The Population Council (NY, USA), do Fundo de Apoio ao Ensino e Pesquisa da Universidade Estadual de Campinas (FAEP/UNICAMP) e do Centro de Pesquisas Materno Infantis de Campinas (CEMICAMP). Agradecemos ainda Maria Helena de Sousa pelo tratamento estatístico dos dados.

\section{Referências}

1. Townsend S. The "new" contraceptive method of breastfeeding. Network 1992; 13: 4-9.

2. McNeilly AS. Lactational endocrinology: the biology of LAM. Adv Exp Med Biol 2002; 503: 199-205.

3. Huffman SL, Labbok MH. Breastfeeding in family planning programs: a help or a hindrance. Int J Gynecol Obstet 1994; 47 Suppl: S23-S32.

4. Kennedy KI, Rivera R, McNeilly AS. Consensus statement on the use of breastfeeding as a family planning method, Bellagio, Italy, 1988. Contraception 1989; 39: 477-96.

5. Winikoff B, Mensch B. Rethink postpartum family planning. Stud Fam Plann 1991; 22: 294-7.

6. Kennedy KI, Labbok MH, Van Look PFA. Consensus statement lactational amenorrhea method for family planning. Int J Gynecol Obstet 1996; 54: 55-7.

7. Diaz S. Determinants of lactational amenorrhea. Int J Gynecol Obstet 1989; 63 [Suppl.1]: 83-9.

8. Kennedy KI, Visness CM. Contraceptive efficacy of lactational amenorrhoea. Lancet 1992; 339: 227-30.

9. Perez A, Labbok MH, Queenan JT. Clinical study of the 
lactational amenorrhea method for family planning. Lancet 1992; 339: 968-70.

10. Labbok MH, Hight-Laukaran V, Peterson AE, Fletcher V, Von Hertzen H, Van Look PF. Multicenter study of the lactational amenorrhea method (LAM): I. Efficacy, duration, and implications for clinical application. Contraception 1997; 55: 327-36

11. Kennedy KI. Efficacy and effectiveness of LAM. Adv Exp Med Biol 2002; 503: 207-16.

12. Hight-Laukaran V, Rutstein SO, Labbok MH, Ballard E. Contraceptive use during lactational amenorrhea. Int J Gynecol Obstet 1996; 54: 101-8.

13. Hight-Laukaran V, Labbok MH, Peterson AE, Fletcher V, Von Hertz H, Van Look P. Multicenter study of the lactational amenorrhea method (LAM): acceptability, utility and policy implications. Contraception 1997; 55: 33746.

14. Labbok MH, Hight-Laukaran V, Peterson AE, Fletcher V, Von Hertzen H, Van Look P. Multicenter study of the lactational amenorrhea method (LAM): efficacy, duration, and implications for clinical application. Contaception 1997; 55: 327-36.

15. Rea MF. Reflexões sobre a amamentação no Brasil: de como passamos a 10 meses de duração. Cad Saúde Publica, 2003; 19 [Supl 1]: S37-S45.

16. Hardy E, Santos LC, Osis MJ, Carvalho G, Cecatti JG Faúndes A. Contraceptive use and pregnancy before and after introducing lactational amenorrhea (LAM) in a postpartum program. Adv Contracept 1998; 14: 1-10.

17. Fisher AA, Laing JE, Stoeckel JE, Towsend JW. Handbook for family planning operation research design. 2nd ed New York: The Population Council; 1991.

Recebido para publicação em 7 de outubro de 2003

Versão final reapresentada em 28 de abril de 2004

Aprovado em 4 de maio de 2004
18. Lebrão ML, Carandina L, Magaldi C. Análise das condições de saúde e de vida da população urbana de Botucatu, São Paulo (Brasil). IV. Morbidade referida em entrevistas domiciliares, 1983-1984. Rev Saúde Pública 1991; 25: 452-60.

19. Hosmer DW, Lemeshow S. Applied logistic regression. New York: John Wiley; 1989.

20. Cervantes Pardo A, Montoya Martinez I, Hernandez Cuevas J, Hernandez Requena C, Talave Lopez J, LopezSanchez S, De Querol M, Canteras Jordana M. Promoción de la lactancia materna. Es efectiva la educación sanitaria? An Esp Pediatr 1993; 39: 529-34.

21. Perez-Escamilla R, Lutter C, Segall AM, Rivera A, Trevino-Siller S, Sangvi T. Exclusive breast-feeding duration is associated with attitudinal socio economic and biocultural determinants in three Latin American Countries. J Nutr 1995; 125: 2972-84.

22. Victora CG, Vaughan JP, Lombardi C, Fuchs SMC, Gigante LP, Smith PG, Nobre LC, Teixeira AMB, Moreira LB, Barros FC. Evidence for protection by breast-feeding against infant deaths from infectious diseases in Brazil. Lancet 1987; 8: 319-21.

23. Bemfam (Sociedade Civil Bem-Estar Familiar no Brasil). Macro Internacional. Pesquisa Nacional sobre Demografia e Saúde: 1996. Rio de Janeiro: A Sociedade; 1997.

24. Rea MF. The Brazilian national breastfeeding program: a success story. Int J Gynecol Obstet 1990; 37 [Suppl 1]: S79-S82.

25. Horta BL, Olinto TA, Victora CG, Barros FC, Guimarães PRV. Amamentação e padrões alimentares em crianças de duas coortes de base populacional no sul do Brasil: tendências e diferenciais. Cad Saúde Publica 1996; 12 [Supl 1]: 43-8. 